\title{
Animation as a Visual Indicator of Positional Uncertainty in Geographic Information
}

\author{
Carsten Keßler and Enid Lotstein
}

\begin{abstract}
Effectively communicating the uncertainty that is inherent in any kind of geographic information remains a challenge. This paper investigates the efficacy of animation as a visual variable to represent positional uncertainty in a web mapping context. More specifically, two different kinds of animation (a 'bouncing' and a 'rubberband' effect) have been compared to two static visual variables (symbol size and transparency), as well as different combinations of those variables in an online experiment with 163 participants. The participants' task was to identify the most and least uncertain point objects in a series of web maps. The results indicate that the use of animation to represent uncertainty imposes a learning step on the participants, which is reflected in longer response times. However, once the participants got used to the animations, they were both more consistent and slightly faster in solving the tasks, especially when the animation was combined with a second visual variable. According to the test results, animation is also particularly well suited to represent positional uncertainty, as more participants interpreted the animated visualizations correctly, compared to the static visualizations using symbol size and transparency. Somewhat contradictory to those results, the participants showed a clear preference for those static visualizations.
\end{abstract}

\section{Introduction}

Uncertainty is inherent in any kind of geographic information (Couclelis, 2003). It can stem from issues such as measurement errors, the limited precision of the sensors in use, processing errors, or the involvement of lay contributors in the collection of Volunteered Geographic Information, to name but a few examples. At the conceptual level, uncertainty can also go back to vague or ambiguous object definitions (Fisher, 1999); non-specific concepts such as downtown are a prime example of the latter (Montello et al, 2003). As Duckham et al (2001, p. 89) put it, 'no observation of geographic phenomena will ever be perfect'. We adopt the broad definition by Longley et al (2005, p. 128) here, who describe uncertainty as 'a measure of the user's understanding of the difference between the contents of a dataset, and the real phenomena that the dataset are believed to represent'. Several theoretical (Worboys, 1998, Gahegan and Ehlers, 2000, Roth, 2009b) and practical (Williams

\footnotetext{
Carsten Keßler

Department of Planning, Aalborg University Copenhagen, Denmark

e-mail: kessler@plan.aau.dk

Enid Lotstein

Bronx Community College, City University of New York, USA

e-mail: enid.lotstein@bcc.cuny.edu
} 
et al, 2008) approaches have been proposed to address this issue. The effective communication of uncertainty of a dataset to its users through visualization remains a challenge, however, both in desktop GIS and in web-based and cloud-based solutions for geographic information.

In this paper, we address the issue of visualizing positional uncertainty for web mapping. Animated maps offer additional visual variables that go beyond the static variables introduced by Bertin (1973), for which DiBiase et al (1992) proposed the three dynamic visual variables of duration, rate of change, and order. Animation can be useful when other visual variables have already been used to represent other aspects of the data and may also be able to convey a different notion of uncertainty. We have tested the efficacy of different static (symbol size, transparency) and dynamic (a bouncing and a rubberband effect) visual variables, as well as combinations of them in an online experiment with 163 participants. We have measured how fast and accurate participants were able to rate the uncertainty in point objects on a web map. Moreover, we have tested how they interpreted the different visual variables, and which kinds of visualizations they preferred. As such, the goal of this work is to address the 'lack of comprehensive empirical work that attempts to cognitively assess uncertainty visualization and decision-making through a human factors standpoint' (Smith et al, 2013, p. 1).

While the presented approaches can also be employed for the visualization of uncertainty in geographic information in other contexts, they are most easily replicated in web mapping. Data visualization frameworks such as D3.js, ${ }^{1}$ which has also been used in our experiment, now allow for a relatively straight-forward implementation of variation in different visual variables, both static and dynamic. Moreover, the capabilities for animation are still limited in most desktop GIS systems, and not an option on printed maps for obvious reasons. We have therefore conducted and evaluated this study in the context of web mapping and designed the experiment to be conducted in a 'natural' environment for the user, i.e., on their own computer, using their preferred web browser.

The remainder of this paper is organized as follows: The next section provides an overview of relevant related work, followed by an introduction to the different visualization types tested in this research in Section 3. Section 4 discusses the design of the online participants test, followed by an evaluation (Section 5) and discussion (Section 6) of the results as well as concluding remarks in Section 7.

\section{Related Work}

The effective communication of uncertainty in geographic information has concerned researchers in GIScience for almost as long as the modeling of this uncertainty. Davis and Keller (1997) have tested both static and dynamic interactive visualizations of uncertainty in slope stability. They conclude that a careful calibration of such visualizations against user specifications (e.g., of allowable uncertainty) is required, especially in risk assessment. Roth (2009a) conducted an online experiment to reveal potential differences between experts and novices assessing the flood risk at a given location. The participants were shown three floodplain delineations, each with a different degree of certainty, visualized with differently colored outlines. The results indicate that domain expertise is more important for decisions under uncertainty than map expertise. Domain experts with little map use experience were still able to accurately assess the risk, whereas map use experts with little or no domain experience underestimated the flood risk with low perceived assessment difficulty - a 'potential disaster' (Roth, 2009a, p. 42). Riveiro (2016) evaluates a similar setting with novice and expert air traffic operators, where uncertainty about the exact position of

\footnotetext{
${ }^{1}$ See https://d3js.org
} 
aircrafts was visualized by circle size. Contrary to Roth (2009a), this study does not find significant differences in performance between the two groups.

Fisher (1993) was among the first to use animation to visualize uncertainty. He used 'flickering' colors on a soil map to represent the certainty of the correct classification of a pixel: the longer the pixel was shown in a color, the higher the certainty that it has been classified correctly. Ehlschlaeger et al (1997) developed animations of elevation surfaces that reflect the uncertainty in elevation measurements. The animations would alternate between different possible realizations of the surface, whereas steps between the different realization scenarios were generated by interpolation. While these two papers proposed animation techniques without having them tested by users, Evans (1997) performed a usability study of a flickering technique to visualize uncertainty. The participants found the flickering on land cover maps a useful, albeit somewhat annoying means of communicating uncertainty in geographic information.

Concerning the visualization of positional uncertainty in point data, McKenzie et al (2016) showed in a recent experiment that the kind of visualization employed does have an effect on the perceived positional uncertainty. Between four different static visualizations shown to participants - a gaussian blur and a solid circle, both with and without a centroid point - the participants responded fastest and most accurate to the solid circle without centroid. Moreover, the test results show that they employed different heuristics depending on the visualization used.

The literature discussed in this section is limited to the work most closely related to the research presented in this paper. Based on advances in both hardware and software libraries to generate animated visualizations, recent research has investigated the use of animation for visualizations in air-traffic analysis (Buschmann et al, 2016), emergency management (Wang et al, 2017), storm surge flooding (Reyes and Chen, 2017) and soil acidification (Russo et al, 2014), to name but a few examples. Readers looking for more complete reviews of the work that has been done around the communication of uncertainty in geographic information are referred to the broad review papers by MacEachren et al (2005), Kinkeldey et al (2014), and Kinkeldey et al (2017). Readers looking for practical recommendations as to which kind of visualization is suitable for which user group are referred to Senaratne et al (2012), who conducted a usability study with different visualizations of uncertainty and users from different backgrounds.

\section{Visualization Types}

In the experiment conducted for this research, the participants were shown eleven different combinations of four different visual variables to represent uncertainty. We used the static visual variables of symbol size and transparency - described as fog by MacEachren (1992) -, as shown in Figure 1.

For the animated visualizations, we used a 'rubberband' and a 'bouncing' visualization. To the best of our knowledge, this is the first systematic attempt to evaluate the effectiveness of animation to convey information about the uncertainty in point data. These two techniques were used because they both provide a clear indication of the area in which the observation was actually made, both with ('rubberband') and without ('bouncing') a direct indication of the measured location.

In the rubberband visualization, the object is moving away from its measured position at a random angle, and then bounces back into that location. This bouncing movement is repeated indefinitely at a constant rate, with a new random angle at every iteration. The distance for the movement is derived from the uncertainty, i.e., the larger the uncertainty,

the larger the distance. The speed at which the object moves varies with the distance: the further it gets away from its measured location, the slower it moves, before it bounces back 

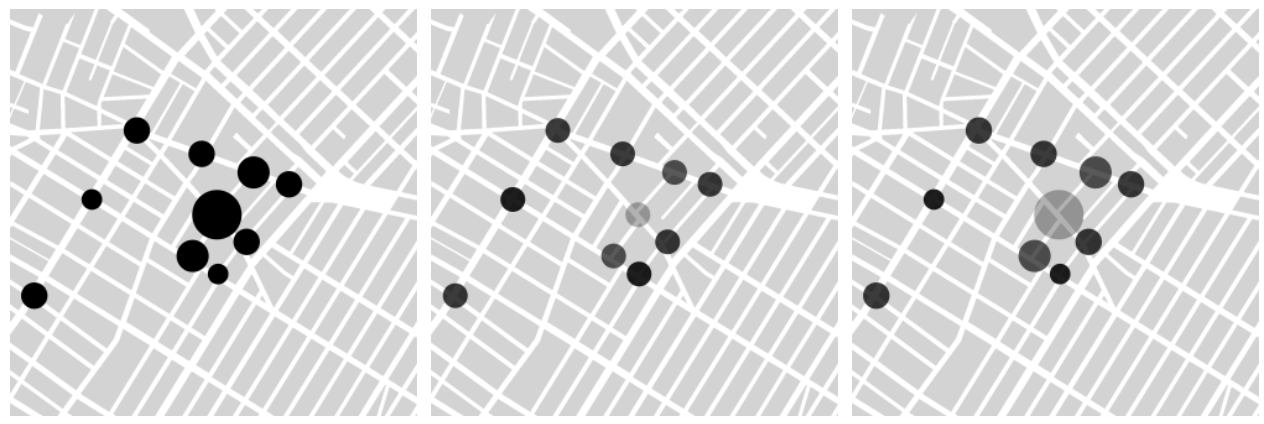

Fig. 1 Static visual variables, from left to right: Symbol size, transparency, and a combination of both.

quickly. Visually, this gives the impression of pulling on a rubberband and then letting go - hence the name. It has been implemented using the elastic easing function in D3.js. ${ }^{2}$

For the bouncing animation, the distance travelled is calculated the same way. The object, however, does not move back to its measured position, it rather moves indefinitely between random positions on the (invisible) circle around the measured position at constant speed. This creates a more 'chaotic' visual effect, as the objects are moving around randomly within that circle; the idea here is to convey the impression that the actual location of the object could be anywhere within this area. Figure 2 illustrates the two types of of animated visualization.
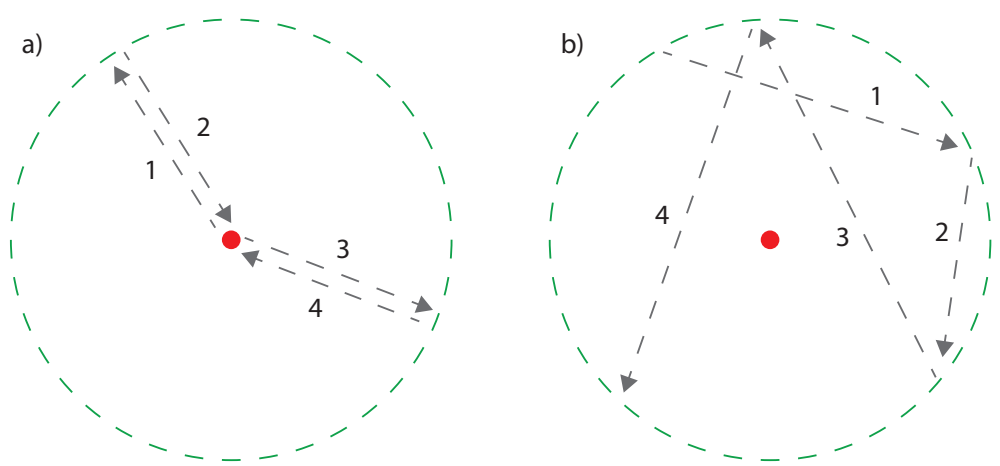

Fig. 2 Illustrations of the rubberband (a) and bouncing (b) animated visualization types. The maximum movement distance (dashed green line) from the measured location (red dot) is a linear function of uncertainty.

The animation techniques used here represent an abstract phenomenon. It is therefore worth noting that they do not fall into any of the types of time (linear, cyclic, or branching) discussed by Harrower and Fabrikant (2008), as the animation is used as a visual effect, and not as a representation of a process in reality. The uncertainty values were mapped to the different static and dynamic visual variables as follows:

- Symbol size: Linear mapping of the range of uncertainty values to circle radii between 3 and 10 pixels, i.e., $\left[u_{\min }, u_{\max }\right] \rightarrow[3,10]$

- Transparency: Inverse linear mapping of the range of uncertainty values to opacity values between $100 \%$ and $30 \%$, i.e., $\left[u_{\min }, u_{\max }\right] \rightarrow[1,0.3]$

- Bouncing and rubberband animation: The size for the circle that marks the maximum distance travelled by an object is calculated the same way as the symbol size above, i.e., $\left[u_{\min }, u_{\max }\right] \rightarrow[3,10]$

${ }^{2}$ See https://github.com/d3/d3-ease\#easeElastic. 
The minimum radius ( 3 pixels) and opacity (30\%) were set to make sure that the objects do not become too small and/or transparent and can still be easily recognized by the participants. The maximum radius was chosen pragmatically to minimize overlap between neighboring points. All visualizations were kept in gray scale colors, including the base map. This was done in order to prevent the participants from imposing any kind of interpretation onto the color choice. Moreover, this excluded any potential effects of color blindness on the results. A minimal style for the base map without any labels was used to prevent any influence of the area shown on the results. Table 1 provides an overview of the test sequence, which consists of 11 pages, each showing a different combination of visual variables.

Table 1 Overview of the eleven different visualizations shown during the test.

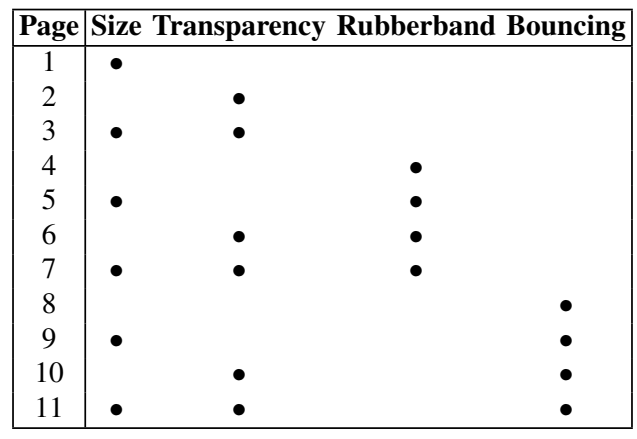

\section{Test Design}

The visualization types discussed in the previous section were shown to a group of participants in an online test. ${ }^{3}$ This section describes the goals of the test, the test sequence and the data used to generate the visualizations discussed in the previous section.

\subsection{Goals}

The goal of this test was to evaluate the efficacy of the visual variables of size, transparency, and two different kinds of animation, as well as combinations thereof in an online test. More specifically, the following questions were driving the design of this test:

1. What kind of visualization allows the participants to identify the most and least uncertain objects on a map quickly and correctly?

2. What are the participants' interpretations of the different visual variables?

3. What are the participants' preferences concerning the different kinds of visualizations?

In order to answer those questions, we have measured how fast and accurate participants were able to rate the uncertainty in point objects on a web map based on the test sequence discussed in the following subsection.

\footnotetext{
${ }^{3}$ The experiment is available online at http://carsten.io/uncertainty/. The corresponding source code and data produced is available at https://github.com/crstn/UncertD3/.
} 


\subsection{Test Sequence}

The test conducted for this study consisted of three parts. The first part was an introduction that explained the goals of the test to the participants. It also contained a preview of the data that were shown to them in different ways during the test (see Figure 3). This was included to allow the participants to familiarize themselves with the map and the area shown before they start the actual test.

The second part of the test consisted of eleven pages that all looked like the one shown in Figure 4. However, every page showed the data using a different visualization type. Every possible combination of symbol size, transparency, rubberband animation, and bouncing animation was tested in the order shown in Table 1. The order was thus not randomized in order to be able to observe a potential learning effect. The uncertainty value associated with each object was randomized for every page to prevent the participants from identifying the most uncertain object once, and then selecting it again on the following pages. The tasks on the eleven pages alternated between selecting the most and least uncertain object in order to test the visualization types both for maximal and minimal uncertainty. The participants selected the object that they believed to be most or least uncertain by placing a red circle around it and clicking. The circle was chosen as a selection tool because simply clicking the corresponding object is very difficult when the points are moving. The selection circle was larger than the biggest possible diameter for the animation of a point. Therefore, participants could select the point by placing the selection circle around it even when it was moving.

The third and final part of the test consisted of a questionnaire. The corresponding web page showed a small example of every one of the eleven visualizations for reference. Participants were then asked to select:

- the best visualization to identify the most uncertain object;

- the worst visualization to identify the most uncertain object;

- the best visualization to identify the least uncertain object; and

- the worst visualization to identify the least uncertain object.

Moreover, the participants were asked about their interpretation of the different symbol variations. For symbol size, transparency, and movement, they were asked whether they thought that the respective visual variable reflects positional uncertainty, uncertainty of a different attribute, or something else. Moreover, they were asked about their interpretation of the relationship between visual variable and uncertainty, e.g., whether larger symbols reflect more or less uncertainty. They were also asked how they interpreted the combination of different visual variables, i.e., whether they all reflected uncertainty, or just one of them. Finally, the participants were asked to provide some personal information: age, gender, and whether they work with GIS or maps at their job or study.

\subsection{Data}

The data used in the test is originally a GPS track of a car driving through Manhattan and Queens, New York City, which was collected by the enviroCar project (Broering et al, 2015). The uncertainty variable used for the visualization in this test is the accuracy of the GPS signal, i.e., positional uncertainty. The randomization of the accuracy values is performed every time a participant visits a page; that way, no two participants will ever see the exact same version of a page. This excludes any effects of the location of the most and least uncertain objects on the results. The uncertainty values are randomized via JavaScript in the participant's browser after loading the original, unmodified GPS track from the server. Af- 


\section{Testing Visualizations of Uncertainty}

In the next minutes, you will be shown different visualizations of uncertainty. That data you will see is a series of points that could be generated from a GPS, for example, overlaid on top of a simple street map. You will be asked to identify the most or least uncertain of those points based on their visualization, which will indicate uncertainty in different ways.

Please not that this test does not work on touchscreen devices such as smart phones or tablets. Please make sure that you are using a laptop or desktop computer if you would like to participate.

Try moving your mouse pointer on the map. It will turn into a circle. You will be asked to place this circle on the point that you believe is the most or least uncertain on the map, and then click.

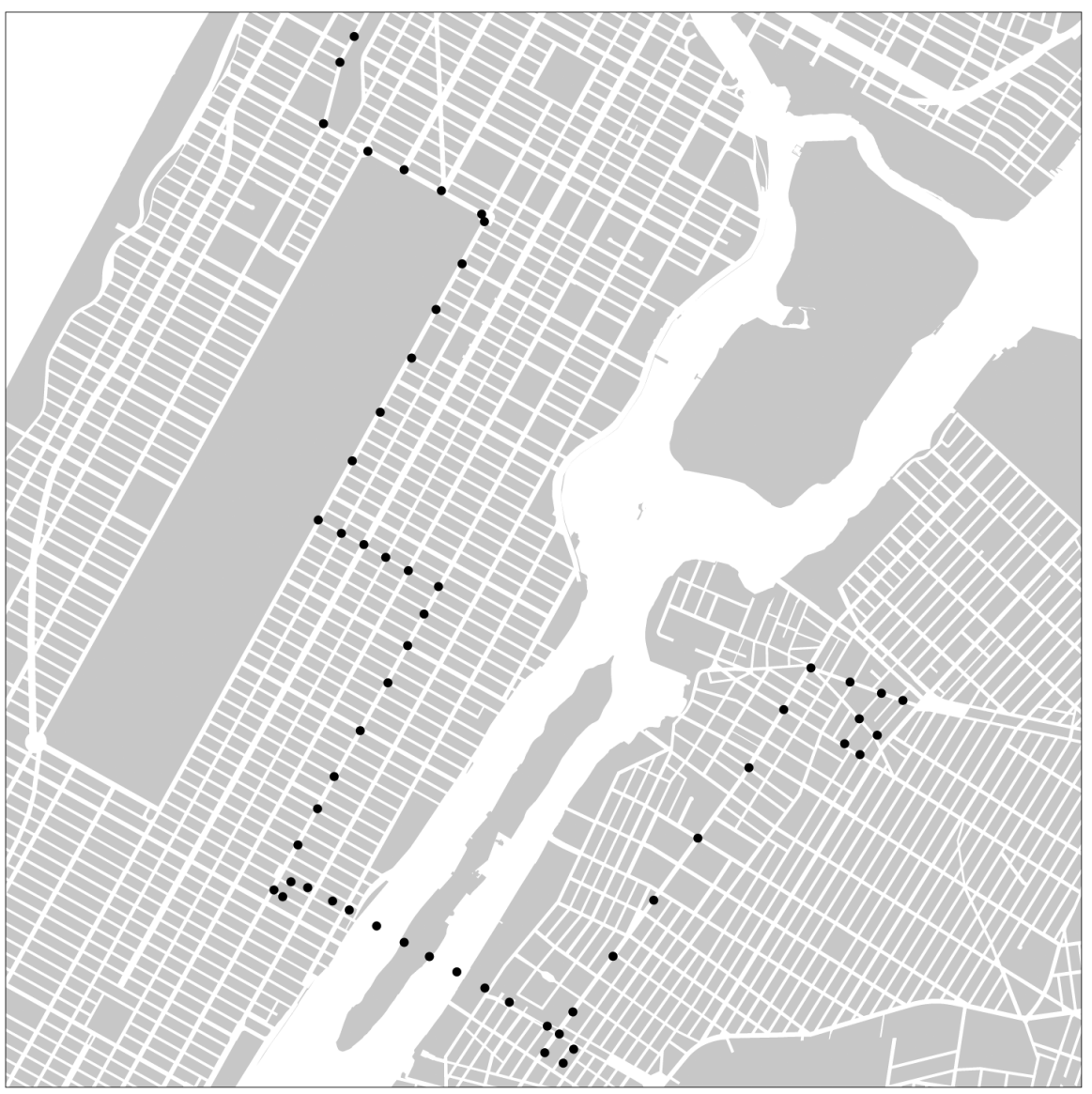

Try to solve this task quickly, but do not rush it - we want you to consider the different points shown and then make a decision. There are no right or wrong answers - please select the point that you believe is most or least uncertain, based on the respective visualization. Participation should not take more than 10 minutes.

Contact: If you have any questions about this experiment, please contact Carsten Kessler.

\section{START}

Fig. 3 Introduction page of the online test. 
Task 3 of 11: Place the red circle on the most uncertain object on the map, then click.

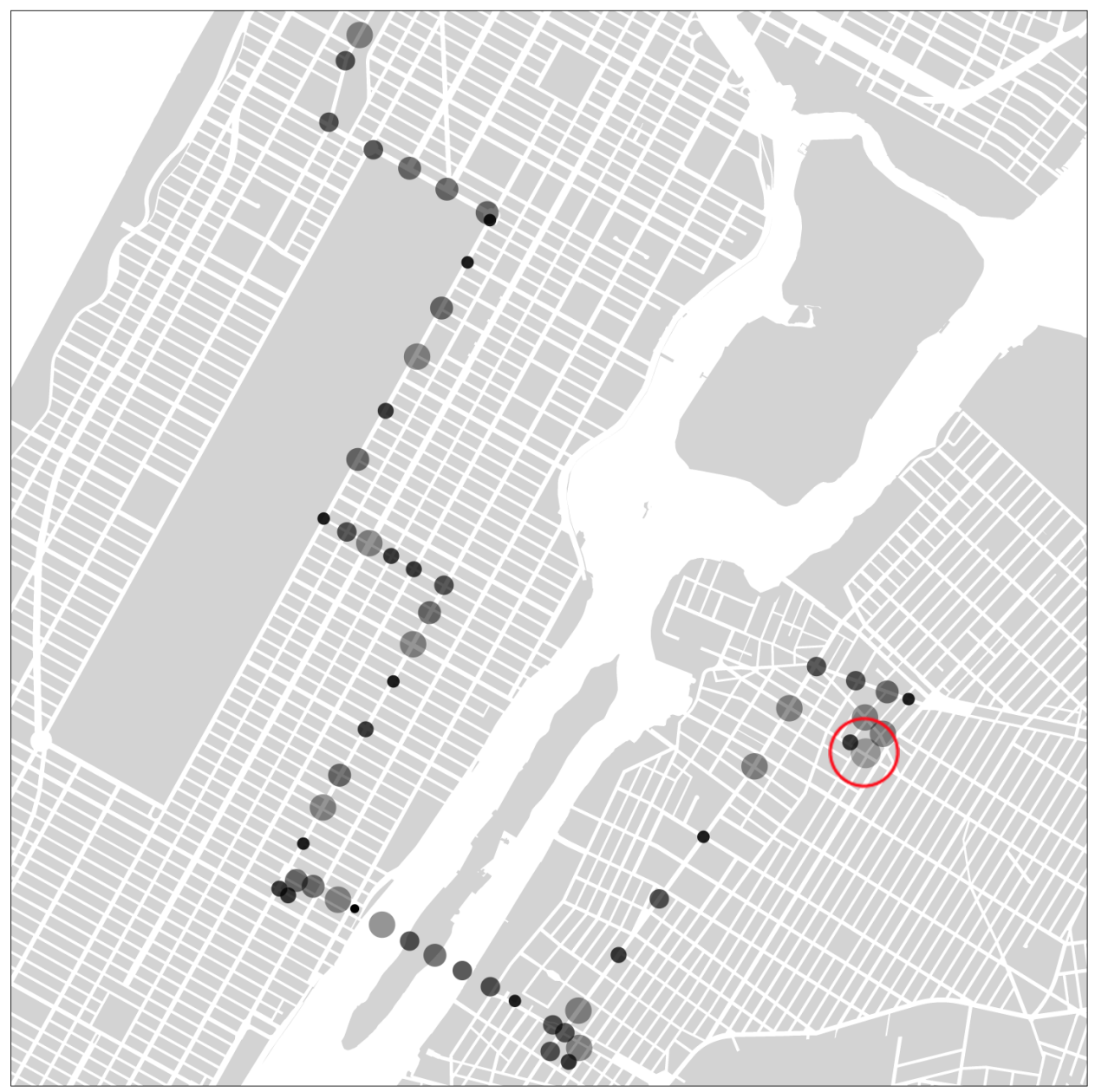

Fig. 4 Example test page with selection circle placed over the most uncertain object.

ter the randomization it is sent back to the server and stored under the participant's session ID and current page number for evaluation.

The random uncertainty values range from 1 to 7 in order to keep them distinguishable from each other for the participants. Values 2 to 6 are assigned completely randomly, i.e., there are generally multiple points in the GPS track that have the same uncertainty value. The lowest (1) and highest (7) values, however, are assigned to exactly one point each. This makes sure that there is exactly one most uncertain and one least uncertain object on every page. These uncertainty values are then used to control the visual variables, as described in Section 3. The GPS track is overlaid on top of a simple base map of the city blocks in New York City, which is intentionally kept very minimalistic to prevent it from driving the participants' attention away from the GPS track. Both the base map and the different visualizations of the GPS track are kept in grayscale to exclude any effects of color choice or color blindness in the participants. It is worth noting that besides the information on the start page (see Figure 3), no additional information about the objects on the map was provided to the participants. A legend or any other further information were intentionally omitted, as one of the goals of the test was also to determine if animation is intuitive as a visual indicator of positional uncertainty. The corresponding questions at the end of the test 
could only be meaningfully asked if the participants did not know what the different visual variables represent.

\section{Evaluation}

This section evaluates the test results with respect to the three research questions from Section 4.1.

\subsection{Participants}

The test was advertised on social media and different mailing lists at the end of October 2016. The tweet announcing the test was retweeted by more than 100 Twitter users, which motivated 163 participants to complete the test within the following ten days. The participants remained anonymous and did not receive any compensation. Based on their responses on the final questionnaire, their mean age was 36 years $(\sigma=10.9)$. The majority of the participants were male (103 or 63\%), 57 were female (34\%), 1 selected other gender, and 2 did not disclose their gender. 133 (82\%) stated that they work with maps, GIS, etc. at work and/or college, whereas $30(18 \%)$ did not. While Twitter did prove very useful for participant recruitment, this overrepresentation of map literate participants is most likely a result of the authors' filter bubble on social media.

\subsection{Visualization Efficacy}

In order to answer research question 1 - What kind of visualization allows the participants to identify the most and least uncertain objects on a map quickly and correctly? - we have analyzed the response times and locations where participants placed the red circle on the map to select the most/least uncertain object. Response times were measured in milliseconds, representing the time that passed from showing the map to the participant until they clicked to submit the location of their choice. The location was measured as the center of the red circle in geographic coordinates.

The density map in Figure 5 has been generated from the locations of all 1,793 locations selected by the 163 participants over 11 pages each. It shows that some of them clicked on locations away from the GPS track, with some of them concentrating in the bottom left of the figure. We have still included these outliers in the analysis because it is not possible to tell whether those participants did not understand the instructions, or whether they clicked these locations for some other reason, e.g., accidentally or because they were not able to identify the most or least uncertain object.

The boxplots shown in Figure 6 give some indication as to which visualization allowed the participants to identify the most and least uncertain objects most confidently and most quickly. Both plots show all 11 test pages in the order given in Table 1, i.e., in the same order they were shown to the participants. The task for the participants was to identify the most uncertain object on all uneven test pages (light blue boxes in the figure), and the least uncertain one on the even test pages (yellow boxes in the figure). Therefore, the 'correct' answer for tests $1,3,5, \ldots$ would be the object with uncertainty value 7 , and for tests 2,4 , $6, \ldots$ the object with uncertainty value 1 .

The boxplot on the left of Figure 6 shows the distribution of the uncertainty values of the point from our GPS track that was closest to the location selected by the user by placing the red circle. It shows that the boxes all have the right tendency -i.e., they are closer to 7 


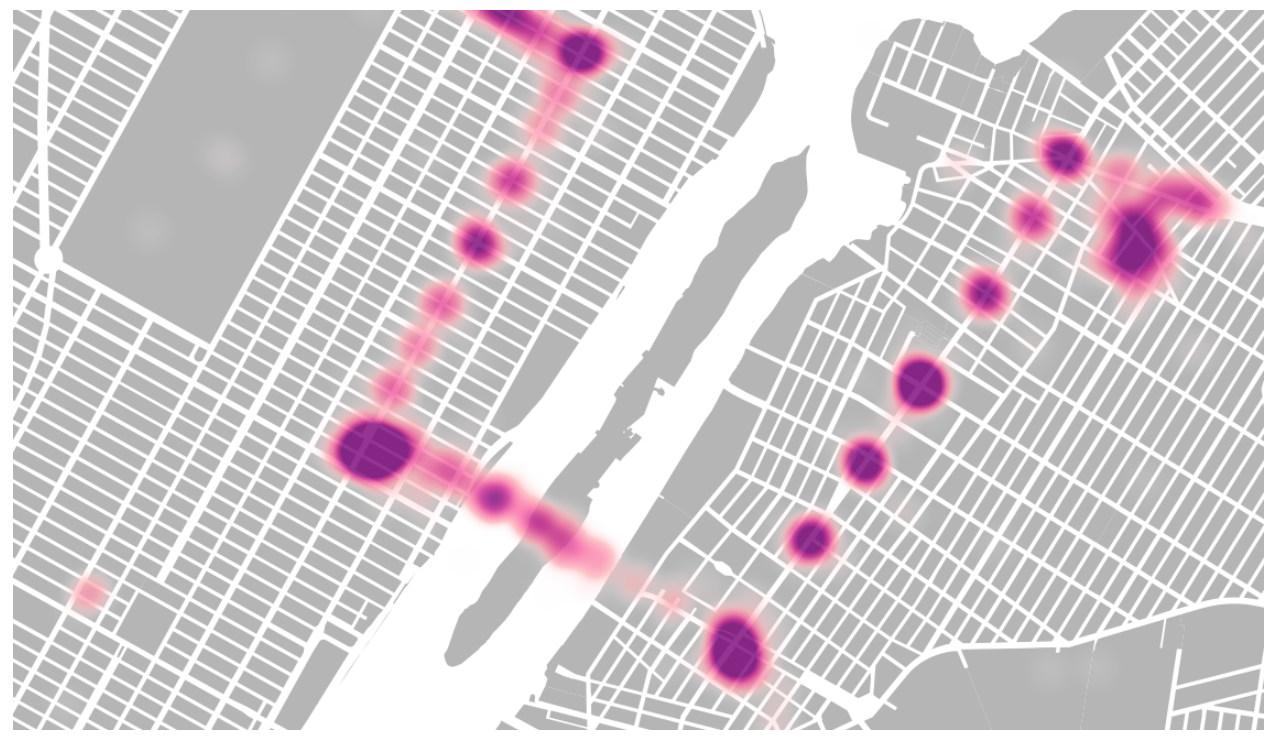

Fig. 5 A density map of the participants' clicks.

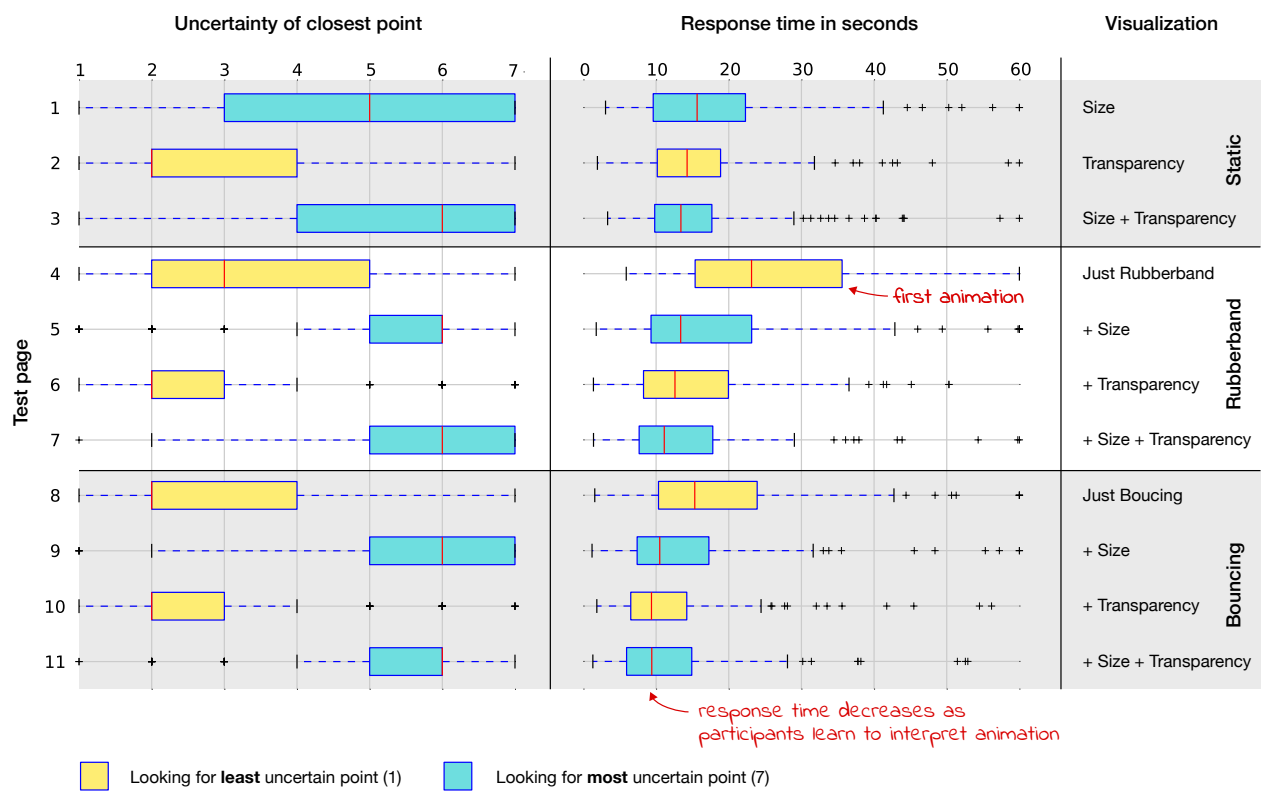

Fig. 6 Box plots of uncertainty of the closest point to the participant's click (left) and the response times (right) for each of the 11 test pages. The red line indicates the median and the whiskers are placed at 1.5 times the interquartile range.

for the tests looking for the most uncertain object, and closer to 1 for the tests looking for the least uncertain object. The fact that the median is not on the 'correct' result for any of the 11 tests can most likely be attributed to the fact that participants could not distinguish between the visualizations of two adjacent uncertainty values ( 1 and 2, for example), and therefore selected an object with value 2 instead of 1 (or 6 instead of 7). The distribution of the results for the selected uncertainty values (shown in the left half of Figure 6), however, shows that the participants were overall more consistent in their judgments on the pages that used animation and at least one other visual variable (pages 5, 6, 10, and 11), as these have the smallest interquartile range. In comparison to the other visualizations, this also means that fewer participants selected uncertainty values that were far off from the correct result. 
Somewhat counterintuitively, the interquartile range is largest for pages 1 to 3 , which were limited to static visualizations. These results indicate that animation may help participants judge the uncertainty of an object correctly if it is used in combination with symbol size and/or transparency.

Concerning the response times ${ }^{4}$ shown in the right part of Figure 6, one apparent outlier is page 4 , where the median response time ( 23.1 seconds) is more than 10 seconds higher than the average median response time on all other pages ( 12.5 seconds). This can be attributed to the fact that page 4 was the first page in the test where the participants encountered an animated visualization, which apparently took them some time to understand. After that, however, the response times are very similar to the response times for the static visualizations on pages 1 to 3 , and even declining towards page 11. This speaks for a learning effect, allowing participants to respond slightly faster after solving several different tasks.

We also tested for correlation between response time and (a) distance from the placed circle to the correct object (with value 1 or 7 , respectively), and (b) difference in uncertainty value between the correct answer and the point closest to the circle (along the lines of the left box plot in Figure 6). Notably, there is no correlation whatsoever between these variables, i.e., the judgments made by participants who only looked at a test page for 5 to 10 seconds were as good as those who took much more time. This still applies if the outliers shown in the density map (Figure 5) are excluded from the analysis.

\subsection{Participants' interpretation}

Research question 2 - What are the participants' interpretations of the different visual variables? - can be answered by evaluating the questionnaire at the end of the test, which explicitly asked for their individual interpretations. Figure 7 shows the counts for responses to the question which kind of uncertainty size, transparency, and animation represented: positional uncertainty, uncertainty in another attribute, or something else. As the bar chart shows, $79 \%$ of the participants interpreted movement as an indicator for positional uncertainty, whereas both symbol size and transparency were significantly more often interpreted to reflect uncertainty in another attribute, or something else. That indicates that animation seems to be an intuitive way to visualize positional uncertainty, and, most notably, more intuitive than the traditional static visual variables of symbol size and transparency.

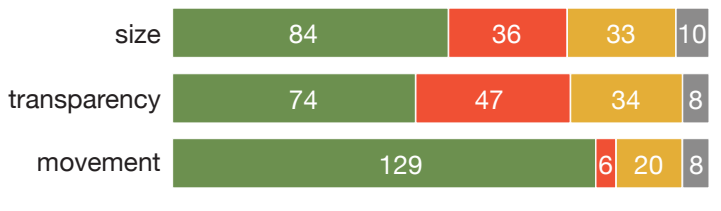

position

other attribute

something else

no selection

Fig. 7 Interpretation of different visual variables.

Figure 8 shows that the relationship between the visual variable and the underlying data was interpreted correctly in most cases: more uncertainty was represented with larger symbols, more transparent symbols, and more movement (i.e., a linear relationship). However, movement also seems to be the most intuitive visual variable in this case, as $91 \%$ of the participants interpreted the relationship correctly. Transparency and especially symbol size

\footnotetext{
${ }^{4}$ Some of the participants had single pages open for several minutes; since it is more likely that they answered the phone or went to get a coffee instead of actually looking at the test page for such a long time, all values above 60 seconds were set to 60 seconds.
} 
were more often interpreted to follow a reverse relationship (i.e., more uncertainty being visualized with smaller symbols).

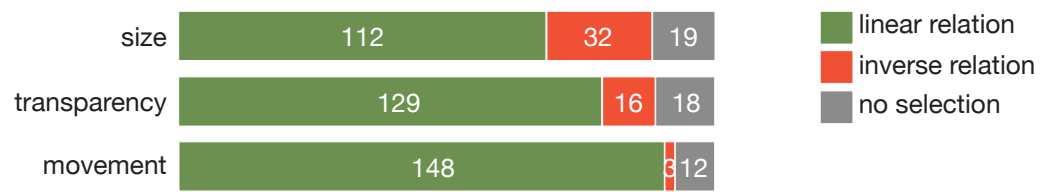

Fig. 8 Interpretation of the relationship between visualization and uncertainty.

The interpretation of two or more visual variables in combination was less consistent. $36 \%$ participants thought that all visual variables represented uncertainty; $17 \%$ thought that just one of the visual variables represented uncertainty, while the others represented something else, and $42 \%$ found them very hard to interpret (5\% did not answer the question). Consequently, while Figure 6 indicates that representing uncertainty with multiple visual variables at once seems to help identify the most and least uncertain objects quickly and consistently, this approach seems to make it more difficult for users to interpret the data.

\subsection{Participants' preferences}

Research question 3 - What are the participants' preferences concerning the different kinds of visualizations? - can also be answered by analyzing the responses to the questionnaire. Four questions were asked about which visualizations the participants found best/worst to find the most/least uncertain object. The responses are summarized in the pie charts shown in Figure 9 and paint a very clear picture: about two thirds of the participants think that the visualizations that only use static visual variables are best to identify the most or least uncertain object on the map. When asked about the worst visualizations for those tasks, the tendency is even stronger: $75-80 \%$ think that one of the visualizations using animation is the worst for the job. These results therefore show a clear preference of static visualizations over animated visualizations, even though the results presented in the previous two sections show certain advantages of using animation to visualize uncertainty.

\section{Interpretation of Results and Discussion}

Participants showed the most consistent behavior with fewer wrong answers for visualizations that used animation in combination with at least one other visual variable. The evaluation of response times, however, shows that animation seems to require an initial learning step, reflected in a significantly longer response time. After encountering the first visualization with animation response times were even slightly lower than for the static visualizations. This gives an indication that a combination of animation with at least one other visual variable may indeed be an effective method to convey information about the uncertainty of geographic information to its users. One potential explanation for these results is that the animated visualizations allow users to inspect parts of the data that are hard to see in a static visualization because of overlapping symbols. In the animated visualizations, these are revealed temporarily as the symbols are shuffled around.

The analysis of the participants' interpretation of the different visual variables, however, shows that animation works most intuitively as a means to communicate positional uncertainty. Even though the participants were never told that the uncertainty in the test did 
The best visualization...
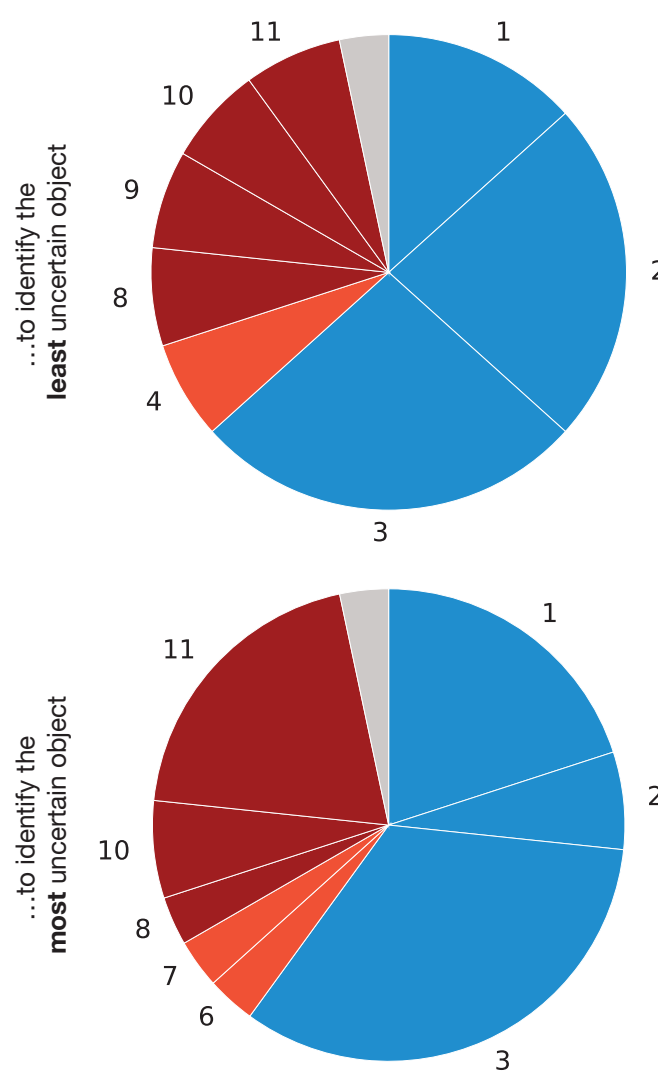

static vis. (1-3)

rubberband vis. (4-7)
The worst visualization...
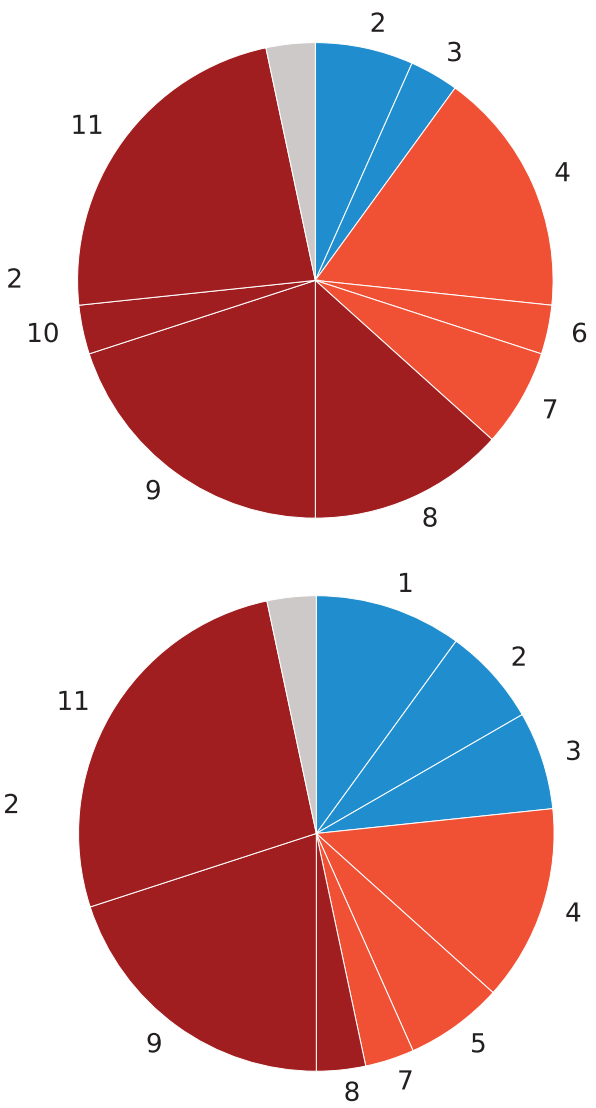

bouncing vis. (8-11)

no answer

Fig. 9 Participants' selection of the best/worst visualizations to find the most/least uncertain object.

indeed reflect positional GPS accuracy, the vast majority interpreted the animated visualizations this way. They also rated the relationship between visual variable and uncertainty value more consistently and more often correct than for symbol size and transparency.

These findings seem to contradict the analysis of participants' preferences, which show that the vast majority preferred the static visualizations over the animated ones, particularly visualization 3, which combines symbol size and transparency. This may be attributed to experimental nature of the animations and that they take some time to get used to. Moreover, the map used in the test did not contain a legend or any other explanations of the meaning of the different visual variables. While this was part of the test design to be able to learn about the participants' intuition, having a legend would most likely have changed this outcome. Designing meaningful legends for animated maps, however, remains challenging (Kraak et al, 1997). One indication that it is possible to use animation with more positive user feedback is the research by Evans (1997), who used a flickering technique to communicate uncertainty. The majority of participants in his study found the flickering helpful.

When interpreting the results of this test, one also has to keep in mind that the majority of the participants can be described as 'map literate'. Therefore, the results may have looked different for a group of participants that do not work with maps on a regular basis. It is worth noting, however, that there were no significant differences in any of the results (response 
time, correctness, participant preferences and interpretation) between expert and non-expert participants. We did not find any significant differences between the female and the male participant groups either, in contrast to previous studies looking at gender differences in the perception of spatial visualizations (Battista, 1990, Maeda and Yoon, 2013).

\section{Conclusions}

We have tested and evaluated the combination of different visual variables to represent uncertainty in geographic information in an online test. To the best of our knowledge, this is the first systematic analysis of the use of animation to represent positional uncertainty in point data. The results give several indications for the effective visualization of positional uncertainty on web maps, especially concerning the use of animation, which allowed participants to solve the tasks slightly faster and more consistently when combined with other visual variables. The participants' preferences are in stark contrast to these findings, as the majority preferred the static visualizations. Future work needs to evaluate whether this rejection of animation disappears as users get accustomed to it. Another avenue for future work is the distinction of different kinds of uncertainty, such as precision, completeness, or currency. Visualizing them in a way so that users can intuitively distinguish them is a research problem in its own right (MacEachren et al, 2005, Andrienko et al, 2010), which may only be solvable for professionals working with geographic information on a regular basis. Finally, the results presented here are limited to point data so far. While the different visualizations can certainly be adapted for lines and polygons, the findings of this paper need to be confirmed for those other geometry types.

\section{References}

Andrienko G, Andrienko N, Demsar U, Dransch D, Dykes J, Fabrikant SI, Jern M, Kraak MJ, Schumann H, Tominski C (2010) Space, time and visual analytics. International Journal of Geographical Information Science 24(10):1577-1600

Battista MT (1990) Spatial Visualization and Gender Differences in High School Geometry Source. Journal for Research in Mathematics Education 21(1):47-60

Bertin J (1973) S'emiologie graphique: Les diagrammes-Les réseaux-Les cartes. GauthierVillars Mouton \& Cie, Paris

Broering A, Remke A, Stasch C, Autermann C, Rieke M, Moellers J (2015) enviroCar: A Citizen Science Plattform for Analyzing and Mapping Crowdsourced Car Sensor Data. Transactions in GIS 19:362-376

Buschmann S, Trapp M, Döllner J (2016) Animated visualization of spatial-temporal trajectory data for air-traffic analysis. The Visual Computer 32(3):371-381, DOI 10.1007/s00371-015-1185-9, URL http://link.springer.com/10.1007/ s00371-015-1185-9

Couclelis H (2003) The Certainty of Uncertainty: GIS and the Limits of Geographic Knowledge. Transactions in GIS 7(2):165-175

Davis TJ, Keller C (1997) Modelling and visualizing multiple spatial uncertainties. Computers \& Geosciences 23(4):397-408

DiBiase D, MacEachren AM, Krygier JB, Reeves C (1992) Animation and the role of map design in scientific visualization. Cartography and geographic information systems 19(4):201-214

Duckham M, Mason K, Stell J, Worboys M (2001) A formal approach to imperfection in geographic information. Computers, Environment and Urban Systems 25(1):89-103 
Ehlschlaeger CR, Shortridge AM, Goodchild MF (1997) Visualizing spatial data uncertainty using animation. Computers \& Geosciences 23(4):387-395

Evans BJ (1997) Dynamic display of spatial data-reliability: Does it benefit the map user? Computers \& Geosciences 23(4):409-422

Fisher PF (1993) Visualizing uncertainty in soil maps by animation. Cartographica: The International Journal for Geographic Information and Geovisualization 30(2-3):20-27

Fisher PF (1999) Models of uncertainty in spatial data. Geographic Information Systems 1:191-205

Gahegan M, Ehlers M (2000) A framework for the modelling of uncertainty between remote sensing and geographic information systems. ISPRS Journal of Photogrammetry and Remote Sensing 55(3):176-188

Harrower M, Fabrikant S (2008) The role of map animation for geographic visualization. In: Dodge M, McDerby M, Turner M (eds) Geographic visualization, John Wiley \& Sons, Ltd, pp 49-65

Kinkeldey C, MacEachren AM, Schiewe J (2014) How to assess visual communication of uncertainty? a systematic review of geospatial uncertainty visualisation user studies. The Cartographic Journal 51(4):372-386

Kinkeldey C, MacEachren AM, Riveiro M, Schiewe J (2017) Evaluating the effect of visually represented geodata uncertainty on decision-making: systematic review, lessons learned, and recommendations. Cartography and Geographic Information Science 44(1):1-21

Kraak MJ, Edsall R, MacEachren AM (1997) Cartographic animation and legends for temporal maps: Exploration and or interaction. In: Proceedings of the 18th International Cartographic Conference, International Cartographic Association, vol 1, pp 253-261

Longley P, Goodchild MF, Maguire DJ, Rhind DW (2005) Geographic information systems and science, second edition edn. John Wiley \& Sons

MacEachren AM (1992) Visualizing uncertain information. Cartographic Perspectives 13:10-19

MacEachren AM, Robinson A, Hopper S, Gardner S, Murray R, Gahegan M, Hetzler E (2005) Visualizing geospatial information uncertainty: What we know and what we need to know. Cartography and Geographic Information Science 32(3):139-160

Maeda Y, Yoon SY (2013) A Meta-Analysis on Gender Differences in Mental Rotation Ability Measured by the Purdue Spatial Visualization Tests: Visualization of Rotations (PSVT:R). Educational Psychology Review 25(1):69-94, DOI 10.1007/ s10648-012-9215-x, $0507464 \mathrm{v} 2$

McKenzie G, Hegarty M, Barrett T, Goodchild M (2016) Assessing the effectiveness of different visualizations for judgments of positional uncertainty. International Journal of Geographical Information Science 30(2):221-239

Montello DR, Goodchild MF, Gottsegen J, Fohl P (2003) Where's Downtown?: Behavioral Methods for Determining Referents of Vague Spatial Queries. Spatial Cognition \& Computation 3(2-3):185-204

Reyes MEP, Chen SC (2017) A 3d virtual environment for storm surge flooding animation. In: 2017 IEEE Third International Conference on Multimedia Big Data (BigMM), pp 244-245, DOI 10.1109/BigMM.2017.54

Riveiro M (2016) Visually supported reasoning under uncertain conditions: Effects of domain expertise on air traffic risk assessment. Spatial Cognition \& Computation 16(2):133-153

Roth RE (2009a) The impact of user expertise on geographic risk assessment under uncertain conditions. Cartography and Geographic Information Science 36(1):29-43

Roth RE (2009b) A qualitative approach to understanding the role of geographic information uncertainty during decision making. Cartography and Geographic Information Science 36(4):315-330

Russo P, Pettit C, Çöltekin A, Imhof M, Cox M, Bayliss C (2014) Understanding Soil Acidification Process Using Animation and Text: An Empirical User Evalua- 
tion With Eye Tracking, Springer Berlin Heidelberg, Berlin, Heidelberg, pp 431448. DOI 10.1007/978-3-642-32618-9_31, URL https://doi.org/10.1007/ 978-3-642-32618-9_31

Senaratne H, Gerharz L, Pebesma E, Schwering A (2012) Usability of spatio-temporal uncertainty visualisation methods. In: Gensel J, Josselin D, Vandenbroucke D (eds) Bridging the Geographic Information Sciences: International AGILE'2012 Conference, Avignon (France), April, 24-27, 2012, Springer Berlin Heidelberg, Berlin, Heidelberg, pp $3-23$

Smith J, Retchless D, Kinkeldey C, Klippel A (2013) Beyond the surface: current issues and future directions in uncertainty visualization research. In: Buchroithner MF, Prechtel N, Burghardt D, Pippig K, Schröter B (eds) Proceedigs of the 26th International Cartographic Conference, International Cartographic Association, pp 1-10

Wang D, Guo D, Zhang H (2017) Spatial temporal data visualization in emergency management: A view from data-driven decision. In: Proceedings of the 3rd ACM SIGSPATIAL Workshop on Emergency Management Using, ACM, New York, NY, USA, EMGIS'17, pp 8:1-8:7, DOI 10.1145/3152465.3152473, URL http: / / doi .acm.org/ $10.1145 / 3152465.3152473$

Williams M, Cornford D, Bastin L, Ingram B (2008) UncertML: an XML schema for exchanging uncertainty. Proceedings of GISRUK, Manchester, UK 44:0-3

Worboys M (1998) Computation with imprecise geospatial data. Computers, Environment and Urban Systems 22(2):85-106 